\title{
eJRIEPS
}

Ejournal de la recherche sur l'intervention en éducation physique et sport

$26 \mid 2012$

Varia

\section{À propos de la dynamique du jeu... en football et autres sports collectifs}

Jean-Francis Gréhaigne et Paul Godbout

\section{OpenEdition}

\section{Journals}

Édition électronique

URL : http://journals.openedition.org/ejrieps/3380

DOI : 10.4000/ejrieps.3380

ISSN : 2105-0821

Éditeur

ELLIADD

\section{Référence électronique}

Jean-Francis Gréhaigne et Paul Godbout, «À propos de la dynamique du jeu... en football et autres sports collectifs », eJRIEPS [En ligne], 26 | 2012, mis en ligne le 01 avril 2012, consulté le 15 janvier 2020. URL : http://journals.openedition.org/ejrieps/3380 ; DOI : 10.4000/ejrieps.3380

\section{(c) (1)}

La revue eJRIEPS est mise à disposition selon les termes de la Creative Commons Attribution 4.0 International License. 
Jean-Francis Gréhaigne* \& Paul Godbout**

${ }^{*}$ GRIAPS. IUFM de l'Université de Franche-Comté. France

** Département d'éducation physique, Université Laval, Québec (QC). Canada

\section{Résumé}

Quel que soit le jeu sportif collectif étudié, une analyse de la dynamique du jeu doit aider à rendre compte du rapport de forces. Contrairement à une affirmation courante, la théorie des systèmes dynamiques, quoique très convaincante du point de vue de la physique, ne peut cependant pas être considérée comme d'une portée générale, résolvant le problème des relations fondamentales entre dynamique et systèmes complexes en sport collectif. Dans cet article, le renversement conceptuel par rapport à une approche traditionnelle techniciste va consister à considérer que rien ne peut être expliqué et réalisé sans une bonne compréhension de la dynamique des rapports d'opposition liant constamment les deux équipes au fil du jeu. La succession et les déformations de l'espace de jeu effectif, l'évolution des configurations du jeu ainsi que la flexibilité inhérente aux formes du jeu constituent des indicateurs privilégiés des conditions fluctuantes de l'affrontement. Avec ces éléments, l'objectif de cette réflexion sera de proposer des modélisations qui permettent de mieux appréhender l'évolution des séquences de jeu.

Ce texte propose une réflexion théorique, principalement à visée épistémique. II permet de discuter la théorie des systèmes dynamiques et son utilisation directe dans le champ de l'étude des sports collectifs, tout en proposant de synthétiser et/ou de créer des outils conceptuels plus pertinents pour analyser des phénomènes évolutifs, complexes.

\section{Introduction}

Après être revenu sur les caractéristiques des jeux collectifs, cet article a pour ambition de présenter une modélisation cohérente et étayée de la dynamique du jeu en sport collectif. A cet effet, il sera discuté la pertinence de l'utilisation de la théorie des systèmes dynamiques pour étudier le jeu en relation avec divers concepts et outils d'analyse de ce que l'on peut appeler « l'école francophone des sports collectifs ».

1. 1. Quelques contributions à la définition des sports collectifs 


\section{eJRIEPS 26 avril 2012}

Les jeux d'invasion sont des sports collectifs qui sont pratiqués entre deux équipes adverses, où les joueurs interagissent directement et simultanément entre eux, sur un territoire commun, pour atteindre un objectif. L'objectif implique généralement que les membres de l'équipe facilitent le mouvement d'une balle ou d'un élément similaire en conformité avec un ensemble de règles, afin de marquer des points. Cela permet de bien les distinguer par rapport au sport d'équipe (cf. huit en aviron) ou aux sports qui relèvent plus du duel tel le tennis en double (Almond, 1986 ; Gréhaigne, Billard, \& Laroche, 1999). En conséquence, dans cet article, nous considérerons donc les sports collectifs comme un ensemble finalisé par un objectif de production de gain du match, bien illustré dans la formule de Jeu (1977) «de l'égalité des chances à l'inégalité du résultat» (p. 131). Mérand (1977) complète ces caractéristiques en présentant les jeux sportifs collectifs comme "une modalité originale d'exploitation de la dynamique des groupes restreints poursuivant un objectif commun» (p. 12). La dynamique de groupes restreints qui s'opposent s'exprime dans la circularité des problèmes à poser et des problèmes à résoudre en vue d'atteindre à la fois l'objectif qui finalise l'action des joueurs partenaires, tout en faisant obstacle aux entreprises des joueurs adversaires. Les multiples possibilités de placement, de déplacement donnent au jeu un caractère d'incertitude, renforcé par le fait que l'initiative change de camp avec le gain ou la perte de la balle. L'idée de groupes restreints qui s'affrontent et l'idée d'efficacité constituent bien l'essence des sports collectifs. Pour Conquet et Devaluez (1986) "l'essence de l'activité est donc un concept qui renvoie au sens; au pourquoi plutôt qu'au comment, au contenu plutôt qu'aux apparences, à la fonction plutôt qu'à la forme» (p. 92). Enfin, pour compléter ce petit tour d'horizon historique, pour Brackenrigde (1979) les sports collectifs sont une lutte pour la conquête d'un territoire avec un ensemble de règles qui incluent des stratégies significatives et des aspects techniques où l'anticipation / coïncidence est de la plus grande importance. La lutte pour la conquête d'un territoire est réglée par un système de score qui symbolise l'importance de la victoire. Le règlement identifie les problèmes et garantit pour l'ensemble des équipes ou des individus l'égalité des chances.

Dans sa thèse en 1989, Gréhaigne affirmait déjà que, pour les sports collectifs, "l'objet de l'action collective, ce qui est transformé entre le début et la fin d'une phase de jeu, est le rapport d'opposition entre les deux équipes afin de marquer dans la cible de l'adversaire tout en protégeant la sienne. Les positions et les mouvements respectifs des joueurs et du ballon sont pris en compte pour caractériser ce rapport d'opposition. L'analyse d'une action en sport collectif nécessite donc le repérage des configurations momentanées, 


\section{eJRIEPS 26 avril 2012}

initiales et finales, ainsi que des transformations successives afin de pouvoir analyser l'évolution du rapport de force, les habiletés motrices utilisées et les choix stratégiques individuels et collectifs » (p. 6).

\section{2. Dynamique et systèmes}

Aussi, quel que soit le jeu sportif collectif considéré, une analyse de la dynamique du jeu doit aider à rendre compte du rapport de forces et des rapports d'opposition. Ici, le renversement conceptuel par rapport à une approche traditionnelle techniciste consistait bien à considérer que rien ne peut être expliqué et réalisé sans une bonne compréhension des rapports d'opposition liant constamment les deux équipes au fil du déroulement du jeu. Analyser, expliquer l'opposition et utiliser cette opposition comme source de tout progrès, constitue une source de références pour concevoir des situations à l'école ou à l'entraînement qui, tout en permettant de faire jouer la réalité de l'opposition, gardent une visée d'apprentissage. Deleplace (1979) affirmait déjà, "nous avons été conduits à démontrer qu'il était nécessaire d'analyser les rapports d'opposition minutieusement et qu'il était parfaitement possible de parvenir à les expliciter, à les formuler et à les systématiser pour leur mise en œuvre consciente et méthodique tant dans l'entraînement ou l'initiation que dans le jeu lui-même » (p. 9). Aujourd'hui tout le monde est à peu près d'accord pour convenir que, pour bien analyser les sports collectifs, il faut recourir à une approche de la dynamique de l'affrontement pour comprendre l'organisation du jeu. Le problème est maintenant de compléter les concepts, et les connaissances pour de nouveaux outils théoriques d'analyse ainsi que des procédés didactiques et pédagogiques rénovés.

La théorie des systèmes dynamiques (cf. McGarry, Anderson, Wallace, Hughes et Franks, 2002, pour une revue dans le sport de compétition ou Davids, Glazier, Araujo, et Bartlett, 2003, concernant le mouvement) désigne couramment la branche des mathématiques qui s'efforce d'étudier les propriétés d'un système dynamique. Cette recherche active se développe à la frontière de la topologie, de l'analyse, de la géométrie, de la théorie de la mesure et des probabilités. La nature de cette étude est conditionnée par le système dynamique étudié et elle dépend des outils utilisés (analytiques, géométriques ou probabilistes). Walliser (1977) appelle système à état (p. 22), un système classique qui évolue au cours du temps de façon à la fois :

- déterministe, c'est à dire que l'état et la sortie à un instant $t$ ne dépendent que des entrées (p. 23); 
- et causale, ce qui traduit le déterminisme local d'un système permettant de suivre son évolution de proche en proche (équilibre, stabilité croissance) (p. 31). Son avenir dépend essentiellement des phénomènes du passé ou du présent.

II faut donc exclure, ici, les systèmes en partie aléatoire, qui relèvent d'une évolution discontinue dans le temps. Les jeux sportifs collectifs avec leurs aspects d'auto organisation et d'organisation, avec des joueurs pensant ou prenant des décisions subjectives (Mouchet, 2003) qui peuvent changer l'évolution du système, semblent plutôt relever de systèmes de type "chaotique ». Ces joueurs ont un comportement infiniment complexe car ils semblent parfois errer au hasard et pas dans d'autres cas. Néanmoins, des attracteurs (une cible, un point d'équilibre sur les phases statiques, un espace ou une configuration) vers lesquels un système évolue de façon irréversible en l'absence de perturbation) caractérisent ces systèmes qui semblent à la fois inclure des lois déterministes et des lois aléatoires, ce qui rend difficile toute prévision à long terme. Contrairement à l'affirmation de Davids, Araújo et Shuttleworth (2005), la théorie des systèmes dynamiques, quoique très convaincante du point de vue de la physique, ne peut cependant pas être considérée comme d'une portée générale, résolvant le problème des relations fondamentales entre dynamique et systèmes complexes en sport collectif. Deux équipes de sport collectif ne sont pas des groupes de particules fonctionnant dans le vide. II est clair qu'il ne suffit pas qu'un système soit composé d'un grand nombre de joueurs pour le voir tendre forcément vers des états d'équilibre. Néanmoins, il doit être possible de suivre l'évolution de l'état d'un système d'affrontement dans le temps car souvent ce système converge vers un état d'équilibre après quelques oscillations ; ce point d'équilibre appelé « attracteur » caractérise simplement un système atteignant un état stationnaire. En sport collectif, nous sommes donc confrontés à une succession d'états temporairement stationnaires au gré des rapports d'opposition au fil du jeu et qui peuvent revenir de façon ponctuelle périodiquement. C'est pourquoi nous préférons parler de la dynamique qu'installe le jeu en mouvement dans un système complexe plutôt que d'appliquer, de façon déductive, la théorie des systèmes dynamiques au jeu. D'autant plus que ce système dit complexe le sera de moins en moins en fonction des informations que l'on peut ou pourra obtenir sur l'état du système et son évolution probable.

En effet, ce qui nous intéresse est plutôt relatif aux conditions permettant une description des propriétés régissant l'évolution du système vers des états d'équilibre ou de déséquilibre, ce dernier étant, sur le fond un équilibre, d'une autre nature en fonction de la cinétique du jeu. Des états finis de configurations connues sont nécessaires pour agir par 


\section{eJRIEPS 26 avril 2012}

rapport à l'infini des configurations possibles, infini qui est subjectif lui aussi. Dans cette perspective, le développement de la compréhension des états de non-équilibre a entraîné de nombreux progrès. Ces considérations montrent que la dynamique se présente parfois sous des caractéristiques entièrement nouvelles. Une telle situation est bien illustrée avec la notion de transition de phase qui, pareillement, n'acquiert un sens précis qu'au moment de la bascule avec l'activation de la situation à double effet (Deleplace, 1966 ; Gréhaigne, 2009 ; Gréhaigne, Godbout, \& Zerai, 2011). Dans le jeu, cette rupture de symétrie introduit la notion de réversibilité par rapport à la phase précédente mais aussi celle d'une éventuelle autre réversibilité dans la phase à venir.

\section{Le jeu en mouvement dans un système complexe}

En poursuivant cette réflexion, nous pouvons dire que les systèmes complexes non linéaires, ou simplement linéaires par moment, savent faire preuve de comportements peu prévisibles qui peuvent même sembler aléatoires. Dans ce cadre, on ne met pas l'accent sur la recherche de solutions précises mais plutôt sur la réponse à des questions comme " existe-t-il des états stationnaires possibles ? ", "le comportement à long terme du système dépend-il des configurations du jeu ? " ou encore "la phase mère du jeu conditionne-t-elle l'évolution du jeu ?». De ce dernier point de vue, un aspect intéressant et innovant est développé par Frencken, Lemmink, Delleman et Visscher (2011) sur les oscillations de la position du centre de gravité du jeu en football et sur la surface occupée dans les jeux réduits (à propos de ces thèmes, on peut, également, consulter Gréhaigne,1992).

\section{1. Configuration du jeu}

Nous définirons une configuration momentanée du jeu comme le schéma formé par la localisation spatiale relative des joueurs des deux équipes sur le terrain ainsi que l'emplacement du ballon à un instant t. Avec les configurations du jeu, un des objectifs sera la description d'états plus ou moins stationnaires du rapport d'opposition. Pour nous, ce sont des configurations du jeu qui, très temporairement, n'évoluent plus avec le temps. On peut penser que certains de ces points fixes sont attractifs, ce qui signifie que si le système parvient à leur voisinage, il va converger vers cet état d'équilibre. De même, on s'intéressera aux aspects périodiques, c'est-à-dire les états du système qui se répètent au bout d'un certain temps. Les configurations prototypiques en sont un exemple particulièrement probant (Caty, Meunier, \& Gréhaigne, 2007 ; Gréhaigne, Caty, \& Marle, 2004). 


\section{eJRIEPS 26 avril 2012}

Dans l'analyse des configurations momentanées du jeu, l'opposition, la continuité et la réversibilité sont trois concepts qui s'avèrent essentiels. L'opposition renvoie à l'affrontement dans un système dynamique spatialement défini par une aire de jeu et un temps limité dans un match où deux sous-systèmes s'affrontent avec des intérêts et des buts opposés. La continuité du jeu et le jeu en mouvement engendrent du désordre provoquant des désorganisations qui tendent à être compensées tant que la défense peut s'adapter. Bien souvent, ce désordre est un désordre apparent où l'on a toutes les raisons de supposer qu'il existe une forme d'ordre caché à qui sait la décoder. L'objet de chacune des actions offensives est bien de provoquer et d'exploiter un déséquilibre du dispositif adverse, de créer l'effet de surprise, l'imprévisible, afin de marquer un but ou un point. Les attaquants doivent s'efforcer de prendre de vitesse la reconstitution par l'équipe opposée d'un équilibre défensif ou d'amener le barrage adverse dans une position critique et ainsi rompre l'équilibre à leur avantage. Enfin, concernant la réversibilité, quand la perturbation n'a pas pu être régulée par les attaquants, cela entraîne la perte de la balle et le passage en défense pour tenter de contrer la contre-attaque avec un bon repli défensif, ce dernier tentant de réinstaurer un état d'équilibre dans le rapport de force.

\section{2. Des états dynamiques}

Le concept d'état dynamique (Gréhaigne, 2009) permet de mieux comprendre comment, à un instant donné, les joueurs sont en déplacement. Ils occupent une position mais cette position est en train d'évoluer car chacun possède une vitesse instantanée différente. Ainsi, l'évolution de la dynamique du système peut alors se modéliser en concevant une évolution discontinue dans le temps. II est à noter qu'en fonction des règles primaires $d u$ sport collectif concerné les degrés de liberté du système sont dissemblables. L'expression degré de liberté recouvre, ici, une notion indiquant la possibilité pour le système d'évoluer dans une direction en partie contrainte à son évolution spontanée.

L'analyse de la dynamique du jeu permet non seulement de percevoir les facteurs contribuant à réguler le jeu mais aussi d'appréhender différents niveaux de régulation et leur interdépendance. Elle conduit également à bien replacer l'activité du joueur et / ou du groupe dans un contexte plus global qui lui donne sa signification. En effet, l'ajustement à la réalité du moment est un processus qui se développe à deux niveaux de façon indissociable : 
- un processus interne qui est l'organisation ou la réorganisation des actions en vue de rétablir la cohérence des réponses du joueur ou du groupe de joueurs à la situation présente ;

- un processus externe qui intègre ces réponses à un ensemble plus vaste représenté par l'affrontement des deux équipes.

Dans le jeu en mouvement, le désordre apparent renvoie souvent à une homogénéité particulière autre que la simple distribution spatiale des joueurs sur le terrain, puisqu'il s'agit d'une distribution sur des niveaux de vitesses. Dans les situations d'opposition, les rapports de vitesses entre les joueurs aboutissent à ce que des états spatialement non homogènes soient compensés et stabilisés. Cela veut dire que ces états apparaîtraient plus homogènes à un observateur qui serait capable de décoder les rapports de vitesses tandis qu'une observation classique mettrait seulement en évidence des aspects hétérogènes parfois structurés par des positions et des formes géométriques. En référence et par analogie aux concepts utilisés par Planck (1941), une telle distribution constitue ce que nous appelons un état dynamique ou une « complexion » du jeu.

Les complexions possèdent en elles-mêmes des possibilités de transformations qui sont à la fois restreintes en fonction des possibles de l'évolution continue du jeu mais importantes si l'on choisit une rupture en modifiant radicalement le mouvement en cours. Elles illustrent le chemin pouvant être parcouru par les joueurs et le ballon dans un laps de temps donné. Certes la circulation du ballon est une chose importante en sport collectif, mais dès que l'équilibre entre les deux équipes se met en place, la circulation des joueurs devient prépondérante. En une seconde la distance parcourue par un ou plusieurs joueurs en fonction de leur vitesse ou de leur accélération peut être importante. Nous proposons une représentation spatiale de ces phénomènes dans les figures 1 et 2 . La modélisation du jeu en mouvement avec les notions de secteurs d'action pour les attaquants et d'intervention pour les défenseurs (Gréhaigne \& Bouthier, 1994 ; Gréhaigne, Bouthier, \& David, 1997)) a permis de mieux comprendre l'importance des rapports de vitesse entre les joueurs pour faire évoluer le rapport de forces. Une complexion (configuration dynamique) du jeu est établie à l'aide de la distribution des joueurs et du ballon sur le champ de jeu en fonction de leurs positions, de leurs orientations et de leurs vitesses de déplacement dans un intervalle de temps donné. 


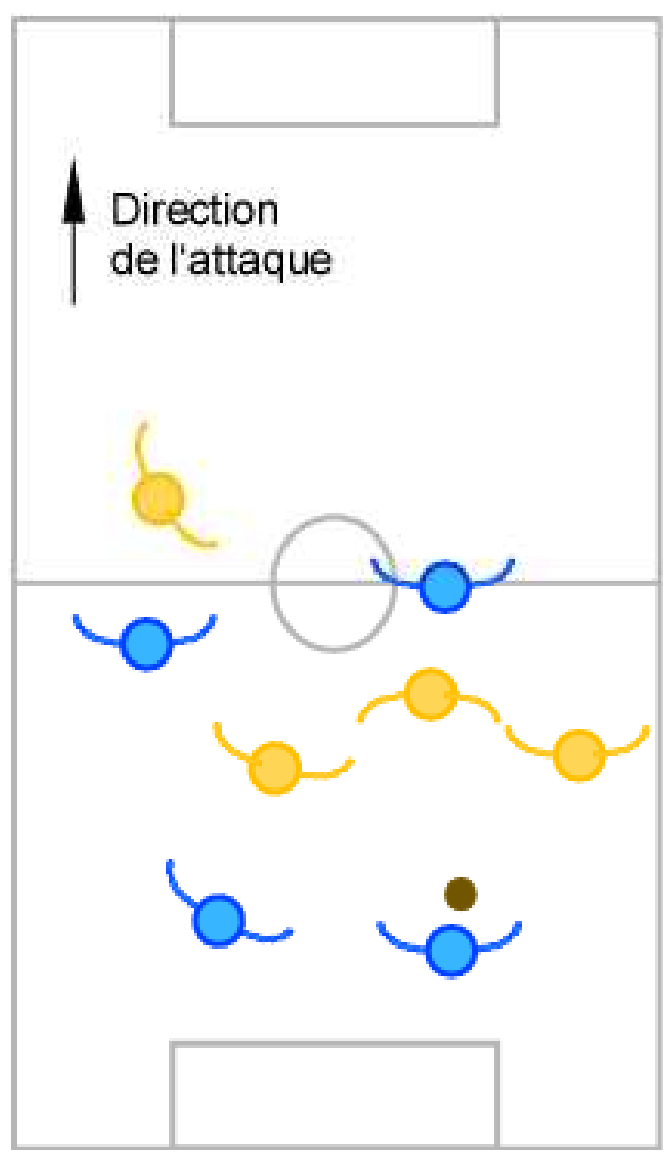

Figure 1. Une Configuration du jeu à T0.

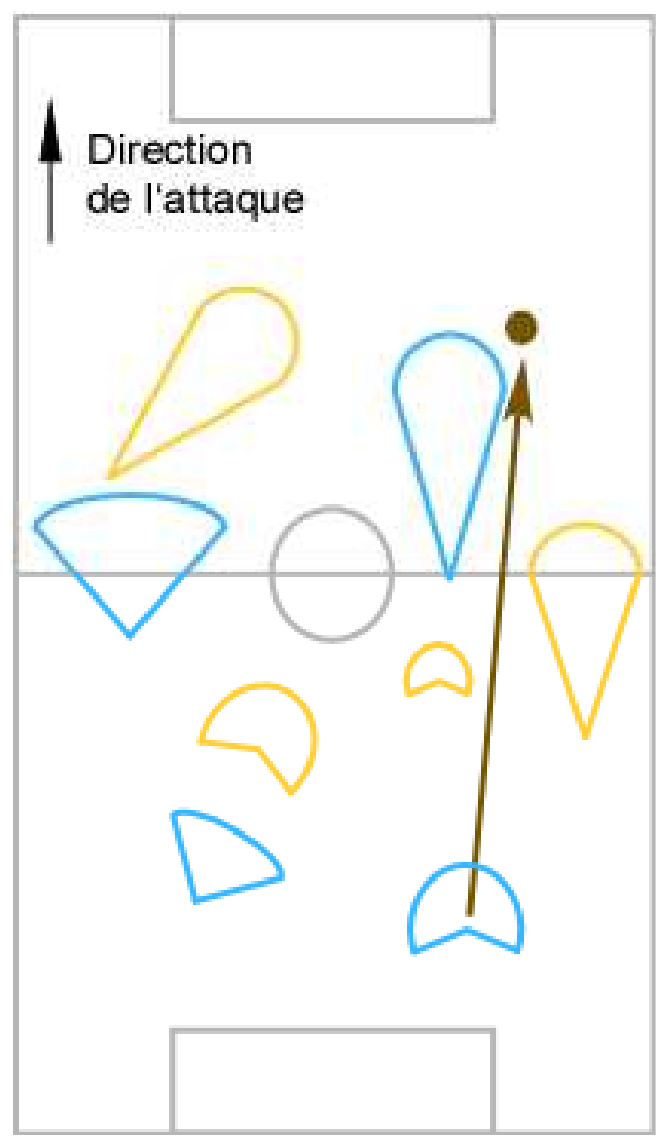

Figure 2. Une «complexion du jeu» à $\mathrm{T}+2 \mathrm{~s}$.

Il est évident qu'en deux secondes, avec des joueurs en déplacement, la complexion du jeu a beaucoup évolué. Certains joueurs ont peu bougé mais d'autres, lancés à pleine vitesse, ont donné une expansion considérable à cet état dynamique. La caractéristique de la circulation des joueurs est qu'elle peut être à la fois très rapide mais aussi entrecoupée d'une succession d'arrêts. Alors, les transitions du jeu entre deux états (deux configurations momentanées du jeu) deviennent des éléments d'observation essentiels pour des décisions appropriées. Barrage / position et poursuite / avance deviennent des éléments premiers de la prise d'information.

2. 3. Les configurations prototypiques

Le temps dont on dispose à l'école ou à l'entraînement pour apprendre en sport collectif est relativement restreint. II faut donc que les joueurs construisent promptement des connaissances et des compétences motrices mais que, une fois construites, ces connaissances puissent servir de base pour d'autres apprentissages plus rapides dans la gestion des configurations momentanées du jeu. Cette notion nous paraît importante car elle est susceptible d'optimiser l'activité des joueurs devant la dynamique du jeu de 


\section{eJRIEPS 26 avril 2012}

mouvement, en leur permettant de caractériser des états momentanés du rapport de forces et leurs évolutions probables.

La méthodologie adoptée pour étudier ce problème a consisté, avec l'aide de bandesvidéo, à sélectionner et analyser des séquences de jeu qui conduisent à un tir cadré ou à un but dans les jeux réduits (Gréhaigne, 2007). La conjugaison de ces critères a permis d'extraire, des séquences vidéo étudiées, des configurations du jeu qui apparaissent le plus souvent avec des joueurs débrouillés. Ces configurations du jeu que l'on retrouve périodiquement et qui sont fondamentalement des états temporairement stables ont été dénommées prototypiques au sens où elles représentent un modèle original, archétype d'un modèle qui se reproduit périodiquement (cf. Gréhaigne, 2007 ; Moniotte, Nadeau, \& Fortier, 2011 ; Zerai, 2011). La connaissance disponible de ces configurations permet aux joueurs de construire des prototypes par catégorisation de formes géométriques, de classes de propriétés d'objets et enfin des catégorisations de relations temporelles en vue d'être plus efficaces en jeu. Ces configurations sont momentanées et au fil du jeu, elles s'enchaînent les unes après les autres. Néanmoins, pour les décrypter, il faut des repères, des images opératives qui permettent de proposer une réponse rapide à la situation perçue. Quelques signes permettent d'identifier rapidement une situation de jeu et son devenir. De ce fait, attirer l'attention des joueurs sur des points clés peut servir avant l'action dans la façon de comprendre, d'organiser, de préparer, et de répondre rapidement au besoin du jeu ou du geste. En conséquence des modèles de jeu peuvent être visualisés avec des manipulations d'images susceptibles de faire réfléchir les sujets sur comment faire : en un mot, comprendre pour réussir. A cet effet, dans les vingt dernières années, la notion d'analogie est devenue un centre d'intérêt majeur pour les cognitivistes et cela a produit de nombreuses recherches (Gentner, Holyoak, \& Kokinov, 2001 pour une revue). II s'agit de tirer des conclusions nouvelles en s'appuyant sur des ressemblances entre deux choses et ce processus intervient dans la pensée quotidienne, l'apprentissage et la résolution de problèmes. Une fois le lien établi, une nouvelle réponse peut être élaborée à partir d'une ou plusieurs réponses déjà construites. De ce point de vue, les matrices offensives et défensives permettent la synthèse d'éléments dans un ensemble ayant comme fondement le mouvement général.

\section{4. Les matrices}

Deleplace $(1979,1994)$ propose, comme première forme de représentation, la notion de système de matrices. Les matrices (au sens génétique du terme c'est-à-dire la source du savoir) " constituent un système cohérent de représentation mentale de la totalité de la 
logique du jeu (p. 21) ». C'est une véritable systématique des décisions tactiques en jeu. Une matrice d'action a pour but d'enserrer la complexité entière du jeu dans un système formé du plus petit nombre possible d'axes fondamentaux mais articulés entre eux dans une unité logique fonctionnelle forte. Ce système doit permettre de redéployer la compréhension du jeu, à la demande, en pleine action dans n'importe lequel de ses axes fondamentaux.

Dans ce contexte, la matrice défensive est l'organisation collective de la défense à la fois la plus simple et la plus générale pour être en mesure d'enrayer, quels que puissent être sa forme, son déploiement et ses rebondissements successifs, le mouvement offensif tenté par l'adversaire momentanément en possession de la balle. À l'inverse, la matrice offensive est d'abord le choix d'une manière de pénétrer dans le système défensif adverse en fonction de sa configuration momentanée.

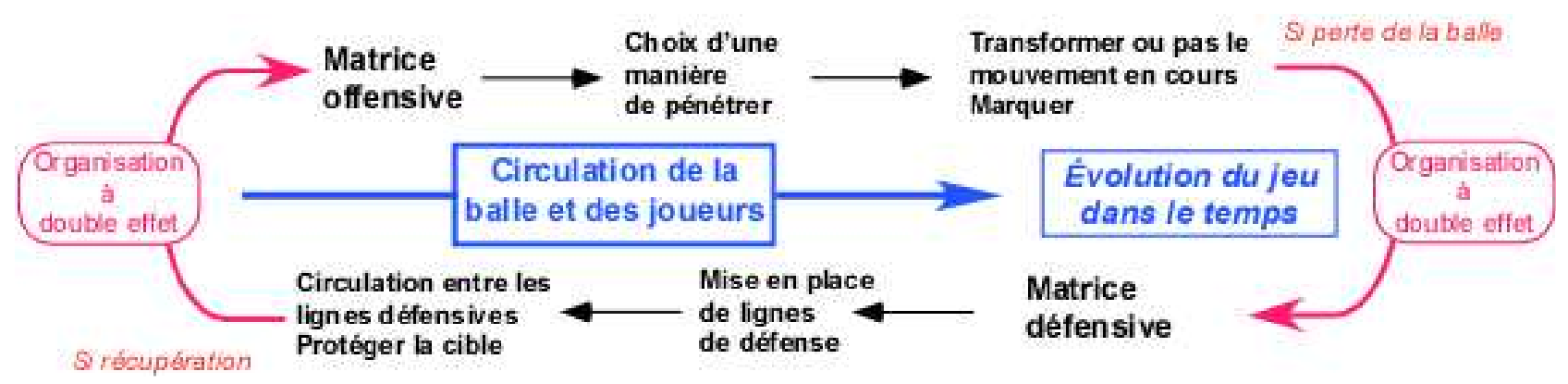

Figure 3. Le jeu et le système des matrices en sport collectif.

Au principe de circulation entre les lignes défensives tout en restant en barrage dans la matrice défensive correspond, dans la matrice offensive, un principe de transformation des mouvements pour prendre de l'avance sur le replacement des défenseurs De ce point de vue, les matrices offensives et défensives sont basées sur des principes d'organisation dynamiques (figure 3). Dans le jeu, la couverture du terrain en défense, ainsi que l'occupation de ce même terrain en attaque, est centrées autour de deux dimensions à tenir qui sont en partie contradictoires: la largeur et la profondeur en relation avec l'étirement (dispersion) et / ou la concentration des joueurs de chaque équipe. En football, cela revient à tenir la largeur de façon constante et la profondeur de façon plus épisodique en fonction de l'organisation du jeu.

2. 5. Organisation et auto-organisation du jeu

Côté organisation, les règles du jeu ont été établies dans le dessein de fournir une structure qui permette le jeu et oriente les actions des pratiquants. Ces règles que l'on nomme premières ou fondamentales constituent les fondements de l'organisation du jeu. 


\section{eJRIEPS 26 avril 2012}

Ces contraintes limitent et régulent les actions des joueurs ; elles sont définies de façon restrictive et non prescriptive, permettant aux joueurs d'expérimenter des réponses. Exemple : au football, on ne peut pas toucher la balle avec les mains (sauf le gardien et sur touche) et non, « il faut toucher la balle avec les pieds »...

Concernant ces règles fondamentales, elles ont trait :

- à la marque, en relation avec les spécificités des cibles du sport collectif concerné qui confèrent aux rapports d'opposition leurs caractères ;

- aux déplacements des joueurs en attaque et en défense, qui découlent de la logique de la marque et la complètent dans le respect de la nécessaire égalité des chances ;

- au degré de liberté d'action sur la balle qui permet de faire vivre de manière spécifique le jeu en favorisant la continuité ou non des mouvements ;

- aux modalités d'engagement physique qui assurent le respect du «parti pris athlétique ».

Pour leur part, les conventions ou règles secondaires permettent de normaliser ou de faire évoluer le déroulement de la pratique. Ce qui les différencie des contraintes, c'est qu'elles peuvent être modifiées sans remettre en cause de façon fondamentale l'essence du jeu. Tout cet ensemble impose des règles d'organisation du jeu au fonctionnement du système d'affrontement.

Côté auto-organisation et d'un point de vue temporel, les phénomènes les plus communs sont soit l'apparition d'états stationnaires multiples, soit l'évolution vers des changements correspondant à un état stationnaire devenu instable à la suite d'une perturbation dans l'évolution du jeu. Ces phénomènes sont connus et interviennent à tous les niveaux de l'organisation du jeu. Tous les phénomènes reposant sur des variations du rythme peuvent être interprétés comme l'apparition d'un ordre temporel, sous forme d'oscillations entretenues. II est remarquable de constater que, quelle que soit la nature des éléments du système de jeu considéré, les interactions non-linéaires entre les éléments donnent souvent lieu à des phénomènes identiques. La raison pour laquelle les phénomènes périodiques sont aussi fréquents en sport collectif tient donc bien à la non-linéarité des processus de régulation. Comme la non-linéarité ouvre la voie à des solutions parfois multiples, on doit s'attendre à des transitions variées entre unités de jeu, ainsi qu'à la coexistence de régimes qualitativement différents. Grâce à ces phénomènes, les systèmes non-linéaires ont la capacité de présenter un comportement complexe qui se traduit souvent par des configurations du jeu diversifiées mais aussi par des configurations 


\section{eJRIEPS 26 avril 2012}

qui reviennent régulièrement. Le problème pour les joueurs devient alors de décoder ces configurations et de les ramener à des prototypes connus.

La situation est fort différente lorsqu'un système opère très loin d'un état d'équilibre. Le système va tenter de répondre à cette instabilité en évoluant vers un équilibre mais, durant cette évolution où le désordre règne, il peut présenter un comportement inattendu pendant un intervalle de temps plus ou moins long. Par exemple, soit l'attaque conserve l'avance prise et le système sera ainsi empêché d'atteindre l'équilibre aussi longtemps que les contraintes seront appliquées et que l'on peut aller vers un but; soit la défense rétablit l'équilibre en revenant en barrage ou récupère la balle. II apparaît clairement que, dans l'organisation du jeu, l'écart à l'équilibre peut donner lieu à des possibilités nouvelles de comportement(s) complexe(s) par une rupture de symétrie. En particulier, on peut repérer ce dépassement du seuil critique d'écart à l'équilibre dans la récupération de la balle à un endroit prometteur du terrain, dans le franchissement de la « ligne d'avantage » ou dans le fait d'amener, rapidement, le ballon à l'avant de l'espace de jeu effectif.

Dans les actions de jeu, comme dans beaucoup de phénomènes où les lois de la physique non linéaire s'appliquent, des perturbations apparaissent à partir d'une certaine vitesse du jeu. II semble exister un seuil critique jusqu'où une vitesse du jeu est tolérable et tout à coup l'adaptation disparaît. En un mot le jeu semble entrer dans un état d'instabilité. On peut retrouver ces événements soit pour le système match (l'affrontement des deux équipes), soit pour une équipe, soit pour un système affrontement partiel. On peut, ainsi, observer à l'intérieur d'une phase de jeu des phénomènes spectaculaires conduisant à un ordre temporel particulier de quelques secondes à la suite d'actions de perturbations (passe longue, centre en retrait, jeu balle en avant) qui s'opposent aux critères habituels de stabilité. En l'absence (supposée) de rupture entre les phases de jeu, on a donc un comportement caractérisé par une répartition uniforme des différentes propriétés du système dans l'espace et dans le temps. Si on provoque délibérément une perturbation pour déclencher un éloignement de cet état, le système développe toujours des mécanismes qui tentent d'amortir cette perturbation. En résumé, la conception du temps et les notions afférentes au temps apparaissent maintenant avec des caractéristiques nouvelles et originales. Ces constatations mettent en évidence tout l'intérêt de la dynamique dans l'étude du jeu car un vieil adage affirme que "face à une attaque en mouvement une défense statique est morte ".

Avec cette thématique les notions de rapport de vitesse, de variation des allures (accélération, décélération) et de perturbations deviennent des contenus importants pour 


\section{eJRIEPS 26 avril 2012}

une didactique des sports collectifs. Dans le jeu en mouvement, la vitesse du ballon et les formes de trajectoires constituent, également, des aspects temporels incontournables pour une bonne formation. Cet enseignement du jeu de plein mouvement requiert donc de revisiter nos habitus d'enseignants ou d'entraîneurs au sens de "structures structurées prédisposées à fonctionner comme structures structurantes " (Bourdieu, 1972 p. 256), afin de proposer de nouveaux objets d'apprentissage.

\section{Sport collectif, dynamique et analyse du jeu}

D'un point de vue pratique, adopter un vocabulaire centré sur la dynamique suppose de bien cerner les termes utilisés. Nous allons donc envisager différentes expressions employées quand on veut parler des conditions de l'affrontement ou de placement et de déplacement dans le jeu.

Le jeu en mouvement crée de la dynamique en fonction de la distribution des joueurs sur le terrain et des rapports de vitesse entre ceux-ci (Gréhaigne et al., 1997). Une façon de représenter les choses consiste à définir les micro-états du système attaque / défense avec pour chaque micro-état l'analyse de la distribution des joueurs sur le champ de jeu en fonction de trois paramètres : leurs positions, leurs orientations et leurs vitesses de déplacement. Cela renvoie à une configuration dynamique temporairement stabilisée qui permet d'anticiper l'évolution du rapport de forces. À un bon niveau, le désordre apparent, au sens physique du terme, désigne alors une homogénéité particulière autre que la simple répartition ou regroupement spatial des joueurs sur le terrain puisqu'il s'agit de distribution sur des niveaux énergétiques à partir de la construction par les sujets d'une transition temporairement stabilisée. Dans les situations d'opposition, ces interactions fondamentalement énergétiques sont liées aux vitesses et aux accélérations / décélérations des joueurs. Cela veut dire que ces déplacements apparaîtraient homogènes à un observateur qui serait capable de décoder les niveaux énergétiques (les quantités de vitesse en interaction) tandis qu'une observation classique mettrait seulement en évidence des aspects hétérogènes mais structurés en rapport avec des positions et des formes géométriques.

Un autre aspect du jeu semble intéressant à explorer du point de vue des systèmes complexes : il concerne les transitions. Les transitions et le jeu de transition renvoient souvent à des configurations où l'on a un peu de temps pour agir car la densité des joueurs est moins importante : l'attaque doit conserver son avance éventuelle et la défense doit revenir en barrage ou y rester. Le recours au jeu long et / ou court transforme 
eJRIEPS 26 avril 2012

rapidement les configurations. Dès que le jeu long est possible, les configurations changent de forme et présentent le plus souvent un jeu en extension. Le modèle en compression se retrouve souvent dans un jeu sur espace stabilisé avec une densité importante de joueurs.

Souvent dans ce jeu en mouvement se succèdent des actions de jeu que l'on nomme classiquement groupé / dégroupé, comme par exemple en rugby, et qui relèvent soit d'une concentration soit d'une dispersion des joueurs. Ainsi, la notion de densité renverrait donc plutôt à un aspect qualitatif car elle dépend étroitement de la qualité des joueurs qui sont engagés dans une action dans un environnement donné. La concentration / dispersion semble plus liée à une approche quantitative en termes de nombre de joueurs engagés dans l'action en cours dans un espace particulier. Compression / expansion pour le mouvement et concentration / dispersion à propos d'états statiques successifs (ou aspects temporels discontinus) semblent fonctionner en étroite symbiose pour qui veut décrypter et anticiper le mouvement dans le jeu en vue de prendre des décisions judicieuses.

Nous sommes bien dans des états dynamiques et c'est essentiellement la mobilité et les déplacements des joueurs qui assurent l'équilibre entre défense et attaque. Cette succession de configurations momentanées du jeu entre l'entrée en possession de la balle et sa perte représente une séquence de jeu.

Ici, la cascade de décisions réduites à un choix binaire ou à la seule étude des dyades appauvrit l'analyse ou semble trop formelle par rapport à l'ensemble des possibles en sportif collectif. C'est en effet faire l'hypothèse que le jeu, en fonction des décisions des joueurs, tend toujours à prendre la configuration la plus probable. On doit en déduire que l'entropie (désordre) liée à la configuration du système est stable. Or la variation de l'entropie d'un système est liée à la probabilité de transition d'une configuration momentanée à une autre, elle-même liée aux probabilités d'apparition d'autres configurations. En particulier, comme le soutient Deleplace (1979), une transformation apparaîtra comme d'autant plus improbable que les probabilités de passage entre deux configurations diffèrent fortement selon le sens de la transformation. Mais l'apparition d'une configuration improbable peut cependant être favorisée :

- par l'existence d'un sous-ensemble de configurations allant d'une configuration probable à une configuration improbable ;

- par la durée de l'affrontement, une situation étant d'autant moins prédictible que l'écoulement du temps est important ; 
- par un événement surprenant (un contre par exemple) qui remet en cause une certaine stabilité du jeu dans le sens d'une augmentation du désordre et ainsi facilite l'apparition d'une configuration inattendue.

La volonté de réduire le désordre dans le jeu au profit d'un ordre extérieur ou d'un modèle prescriptif ne doit donc pas conduire à ne pas prendre en compte l'entropie dans la modélisation du jeu. Aussi, un autre point important à aborder concerne la réversibilité des situations avec le passage de l'attaque à la défense.

\section{Dialectique, dynamique et rapports d'opposition}

Le point de vue développé ici, à propos des rapports d'opposition, conduit, bien souvent, à une analyse en termes contradictoires des configurations du jeu, comme, par exemple, avant / arrière de l'espace de jeu effectif (EJE) ; barrage / poursuite ; étalé / resserré ; défense / attaque en fonction de la possession ou non du ballon ...

4. 1. Un point de vue dialectique

Dans ce type d'approche tactique évoqué ici, de nombreux concepts doivent être considérés de manière contradictoire, donc d'un point de vue dialectique. Parmi les nombreuses significations de la dialectique, nous avons choisi de la définir comme une façon de penser - au sujet des objets ou des idées - qui utilise l'opposition ou la divergence de pensée pour atteindre une synthèse. Le raisonnement dialectique consiste à aller et venir entre des raisonnements contraires et ainsi la contradiction est la clé de toute réflexion critique relative aux problèmes à résoudre. Dans les sports d'équipe, les joueurs peuvent avoir des situations où l'information est incomplète, mais aussi être en présence de configurations où de nombreux éléments, à qui sait les identifier, peuvent contribuer à faire évoluer la séquence de jeu.

En fonction des caractères permanents du jeu en football, nous ajoutons maintenant d'autres éléments pour analyser le rapport de forces afin d'améliorer la compréhension de l'évolution du jeu. II existe donc pour les décisions à prendre : continuité / rupture par rapport à l'action en cours, liée organiquement à continuité / rupture par rapport à la possession de la balle. Ceci entraîne une circularité des problèmes posés aux équipes, au plan de l'organisation du jeu comme au plan de la distribution réelle des joueurs (figure 4). Cette notion de rapport de forces, entre les deux équipes en présence, permet également de mettre en évidence la notion de pression sur tel ou tel maillon du jeu pour rompre l'équilibre des forces en présence dans les affrontements momentanés. Ainsi, on vise à 


\section{eJRIEPS 26 avril 2012}

créer un déséquilibre favorable pour aller marquer un point. Enfin, deux autres aspects sont à gérer contradictoirement :

- soit prendre des risques pour prendre de l'avance sur le replacement défensif mais en n'assurant plus tout à fait une couverture défensive suffisante,

- soit privilégier la sécurité en préservant temporairement une certaine stabilité défensive (pour éviter de prendre du retard) mais en laissant l'initiative du jeu aux adversaires.

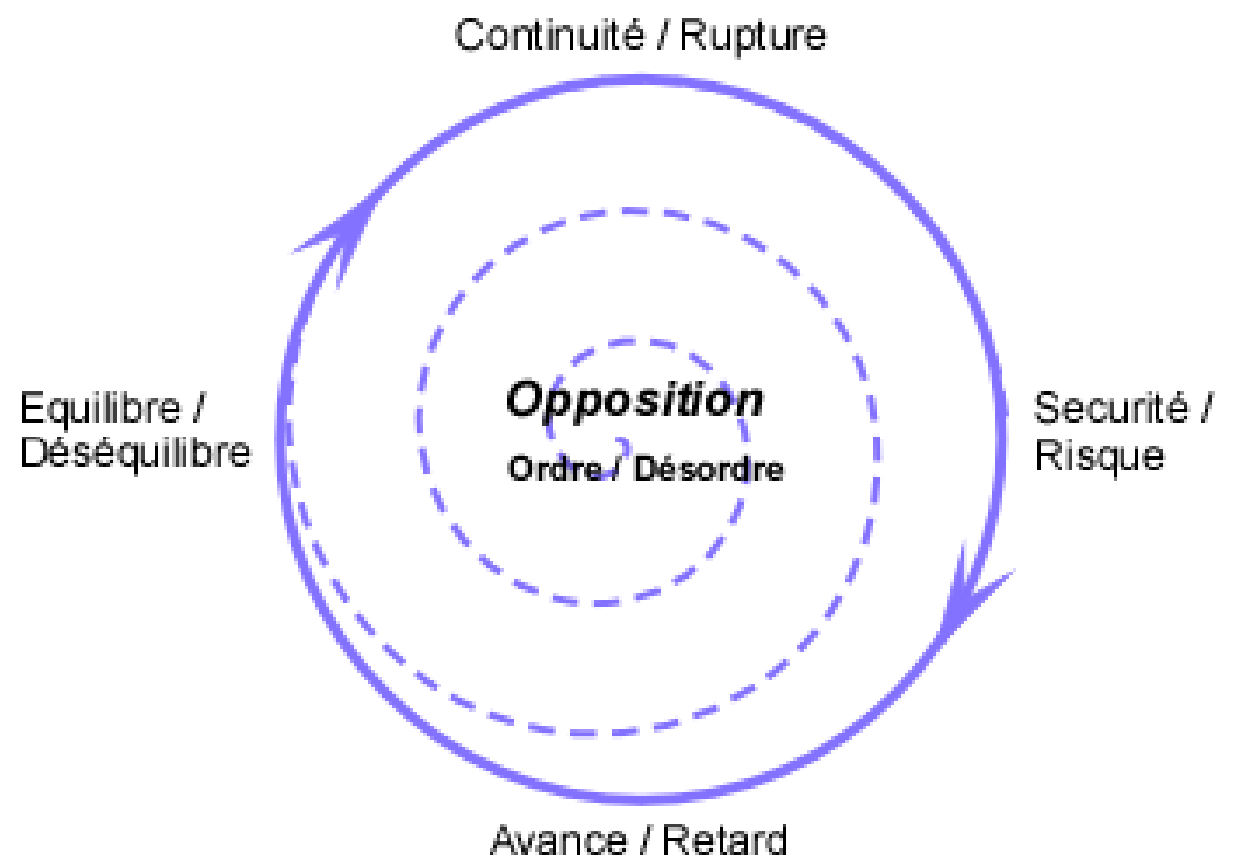

Figure 4. Des concepts pour analyser la situation d'opposition (Gréhaigne, 2011).

L'ensemble de ces notions ne relèvent pas toutes du même plan, mais concourent, chacune à leur manière, à la complexité du jeu. Nous allons, maintenant, poursuivre cette étude avec une réflexion sur la réversibilité du jeu.

\section{2. La réversibilité}

La réversibilité des situations représente un aspect fondamental des sports collectifs en rapport avec le fait que les équipes attaquent et défendent tour à tour, et qu'il y a toujours une part de défense dans l'attaque et une part d'attaque dans la défense (Gréhaigne, Godbout, \& Bouthier, 1999). On peut parler d'une véritable "co-construction "du système attaque / défense, ce système se faisant et se défaisant au gré de l'action.

La réversibilité concerne également les comportements des joueurs quand le rapport de forces est trop déséquilibré. II les fait basculer, souvent, dans un rapport de « dominé "... avec ses conséquences sur le jeu. Par exemple, la possession de la balle en attaque peut 


\section{eJRIEPS 26 avril 2012}

se réduire à une simple mais problématique conservation quand l'adversaire est trop largement supérieur et que le principe de « l'égalité des chances à l'inégalité du résultat » ne peut plus être respecté. Ici l'égalité des chances ne renvoie pas forcément à la supériorité ou à l'infériorité numérique car la réversibilité des comportements existe aussi sous l'effet de la fatigue, de la motivation ou d'une baisse d'attention ...

Nous avons déjà souligné que les configurations du jeu avec leurs formes diverses peuvent apparaître dans diverses parties du terrain en fonction des rapports d'opposition et présenter des dangers variés. Une perte de balle à l'arrière de l'espace de jeu effectif (Mérand, 1977 ; Gréhaigne, 2007) dans son propre camp représente un danger important pour l'équipe qui était en attaque et qui se retrouve en défense dans des conditions délicates. Pour mieux découvrir la dynamique du système, on peut analyser les configurations du jeu en séparant les espaces de jeu effectifs de l'attaque et de la défense et se construire un schéma d'étude du jeu plus dialectique.

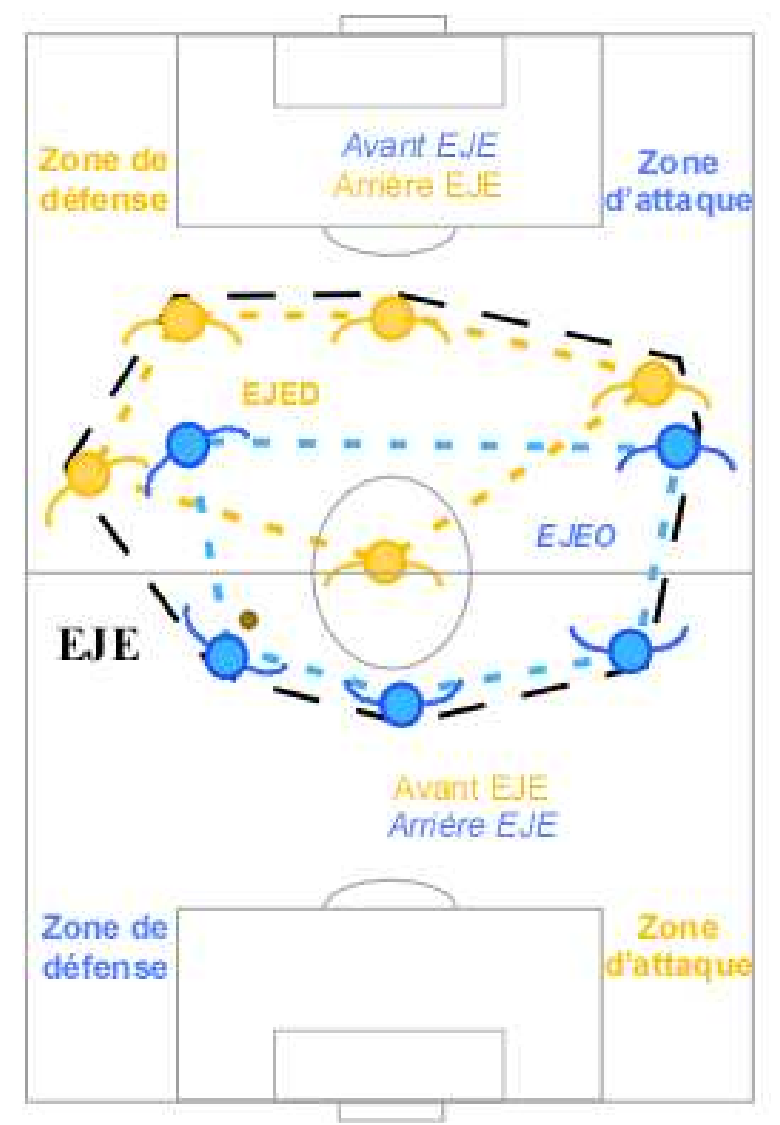

Figure 5. Champ de jeu et espaces de jeu effectif offensif et défensif avec l'exemple du football ; affrontement et réversibilité entre les bleus en attaque et les oranges en défense.

Dans la figure 5 (avec par convention une défense positionnée en barrage), nous illustrons le potentiel offensif des attaquants (en bleu) et défensif des défenseurs (en orange) en 


\section{eJRIEPS 26 avril 2012}

regard de l'espace (largeur / profondeur, emplacement sur le terrain, barrage / poursuite) et du temps (vitesse du jeu, extension / compression, retard / avance). Concrètement, ce schéma d'analyse permet de caractériser la configuration du jeu de la figure 5. L'espace de jeu effectif est au milieu du terrain, la défense est en barrage avec un ballon en arrière de l'espace de jeu effectif offensif à sa périphérie. On est dans une phase de montée da balle par l'équipe bleue avec des EJEO et EJED peu interpénétrés. À cet instant, l'EJED et l'EJEO sont plutôt étalés dans la largeur. Nous sommes dans une phase classique de jeu de transition ou de jeu au milieu du terrain dans le cas du football.

Pour estimer l'effet du temps sur cette configuration, il est nécessaire d'avoir plusieurs états successifs afin d'apprécier la cinématique des actions de jeu. Ce type de travail (cf. Ziane, 2004 pour un exemple en basket-ball) prend en compte les mouvements des joueurs, des équipes et du ballon ainsi que les relations entre ces mouvements et ainsi permet d'interpréter avec précision les variations de configuration des équipes.

II faut rappeler aussi que cette réversibilité est contrôlée par le règlement avec, suivant les sports collectifs, des zones interdites temporairement ou de façon permanente en vue d'équilibrer le rapport attaque / défense. Cela pèse bien sûr sur les rapports d'opposition et induit certains types de jeu.

\section{3. Contraction / expansion}

Une vision dynamique de l'affrontement entraîne la nécessité d'imaginer d'autres concepts pour rendre compte du mouvement. À cet effet, et pour mieux analyser l'étalement des espaces de jeu effectif, nous avons présenté (Gréhaigne, 2009) les concepts d'espaces de jeu effectif en extension et / ou en compression afin de constater ce que pourrait amener cette autre façon d'envisager l'analyse du rapport de forces entre deux équipes (figures 6 et 7 ). 


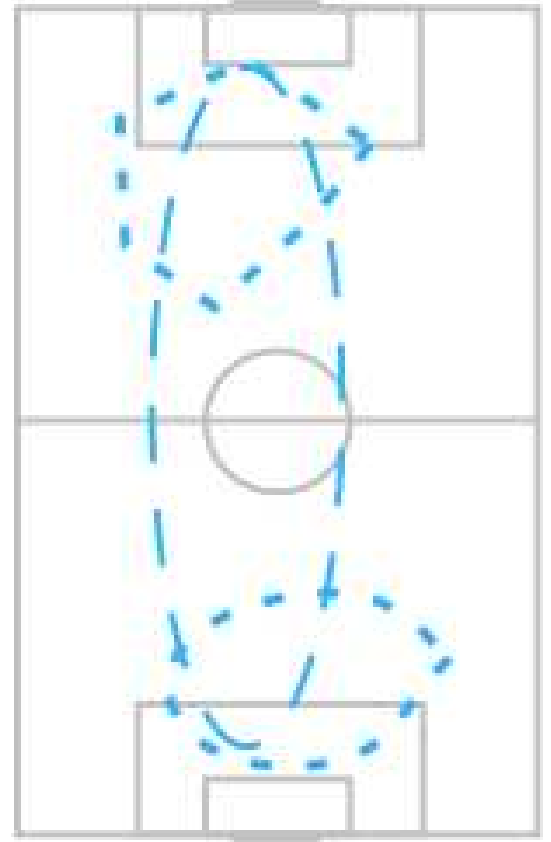

Figure 6. Contraction / Extension

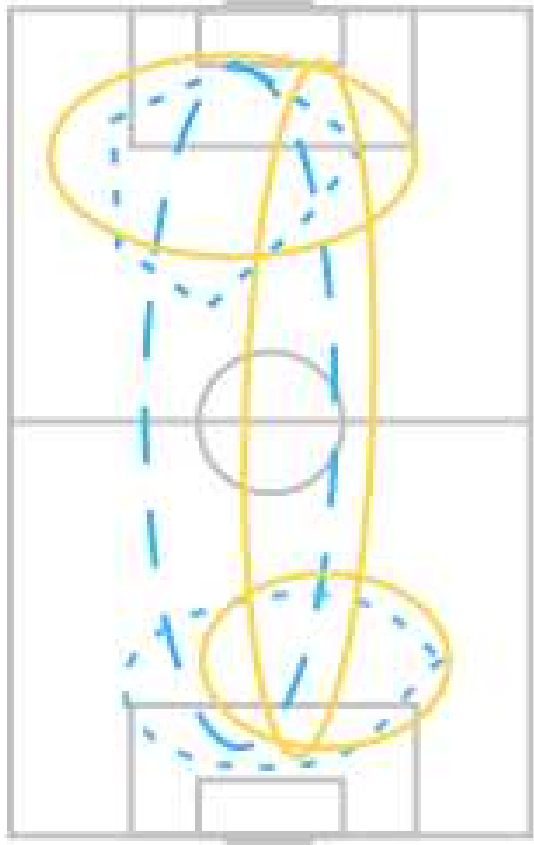

Figure 7. Contraction / Extension / Contraction des deux équipes

Les transitions et le jeu de transition renvoient souvent à des configurations où l'on a un peu de temps pour agir (sauf pour la contre-attaque) car la densité des joueurs est moins importante : l'attaque doit conserver son avance éventuelle et la défense doit revenir en barrage ou y rester. Le recours au jeu long transforme rapidement les configurations et celles-ci présentent le plus souvent un jeu en extension. Le modèle en compression se retrouve souvent dans un jeu sur espace stabilisé près de la cible avec une densité importante de joueurs. Les phases de transition entre un jeu stabilisé dans la zone de défense et ce même jeu stabilisé dans la zone d'attaque sont très importantes à repérer car on va souvent de la compression vers l'extension et vice versa Pour compléter cette réflexion, on peut dire que dans une acception courante, la densité dans le jeu « mesurerait » la dispersion des joueurs sur une surface donnée. Ce serait donc, a priori, une valeur essentiellement spatiale et non numérique si l'on se réfère encore une fois à des états statiques. II existe une relation simple entre dispersion, densité et distance : plus la distance entre les joueurs augmente plus la densité diminue et plus la dispersion augmente jusqu'à une limite où elle deviendrait constante. Les limites de ces phénomènes seraient constituées, sur un continuum, par une répartition uniforme de tous les acteurs sur l'aire de jeu (correspondant à une dispersion maximale de ceux-ci) ou un regroupement en un seul endroit sur le terrain (correspondant à une concentration maximale de tous les joueurs). 


\section{eJRIEPS 26 avril 2012}

Dans la réalité, densité (variable plutôt qualitative) et dispersion / concentration (variables plutôt quantitatives) fonctionnent à l'intérieur de possibilités définies que sont les limites extérieures de l'aire de jeu utilisée. Dans une séquence de jeu, il peut exister, au même moment, une concentration dans certains endroits du terrain et une dispersion dans d'autres. Si l'on joue dans l'axe central, la dangerosité du mouvement en cours peut être appréciée en fonction du temps, des événements du jeu et de la proximité de la cible ; d'où, par exemple, une concentration de joueurs dans l'axe but attaqué / but défendu et une dispersion importante à la périphérie du terrain.

En conséquence, en sport collectif, la densité renverrait donc plutôt à un aspect qualitatif en fonction des zones de jeu utilisées, la concentration / dispersion semblant plus liée à une approche quantitative en termes de nombre de joueurs. Compression et extension pour le mouvement, contraction / expansion à propos d'états statiques successifs (ou aspects temporels discontinus) semblent fonctionner en étroite symbiose pour permettre aux joueurs de décrypter et d'anticiper le mouvement dans le jeu et ainsi prendre des décisions judicieuses. Les principales actions concernant la circulation du ballon et des joueurs peuvent être ainsi inventoriées :

- balle arrêtée dans la contraction ;

- balle arrêtée avec une phase d'expansion ;

- balle en mouvement vers ou dans la contraction;

- balle en mouvement dans une phase d'expansion.

Pour plus de précision, il faut, bien sûr, intégrer à cette analyse les trajets et trajectoires du ballon (dans l'axe ou à la périphérie) pour obtenir une appréciation plus exacte de la situation et du rapport de forces attaque / défense. Après ces précisions sur le mouvement dans le jeu, nous allons en venir maintenant à une proposition de modélisation.

4. 4. Un modèle de la dynamique

Comme nous venons de le voir, l'étude théorique des rapports d'opposition permet de dégager un certain nombre de concepts pertinents qui conduisent à la définition de variables ou d'indicateurs liés de façon plus ou moins compliquée (nombre, rapports, fonctionnalités) à des aspects observables. Cette analyse théorique permet de suggérer, aussi, un certain nombre de relations possibles entre ces indicateurs ainsi que des contraintes aux limites de fonctionnement du système observé.

Aussi, si nous poursuivons un peu plus avant notre réflexion, dans la figure 8, déplacements, contractions et expansions de l'espace de jeu effectif sont au cœur de la conception de notre modélisation. Dans le système «match", chaque équipe est 


\section{eJRIEPS 26 avril 2012}

caractérisée par un taux d'entropie (désordre) qui traduit le degré d'organisation ou de désorganisation du groupe. Révélateur du fonctionnement collectif, ce taux doit néanmoins rester dans des valeurs acceptables pour permettre l'affrontement. Trop d'ordre apparent chez les attaquants impliquerait en effet une tactique aisée de récupération et de contre pour l'adversaire, tandis que trop de désordre induirait des aléas difficilement surmontables par les partenaires.

Dans le système "affrontement partiel » l'interpénétration des sous-systèmes et le fait qu'ils soient en barrage ou à la poursuite avec de l'avance pour l'attaque ou du retard sur le replacement défensif pour la défense va façonner des rapports d'opposition spécifiques. Enfin, les trajets et les trajectoires de la balle fournissent des observables essentiels car, par exemple, en envoyant le ballon dans le dos des défenseurs, une bonne longueur de balle peut mettre l'adversaire en difficulté. Une accélération de la circulation du ballon en diminuant le nombre de touches de balle entre les partenaires constitue, aussi, une option économique, fiable et sécuritaire de progression vers le but adverse (Dugrand, 1989 ; Lemoine \& Jullien, 2008). Le football moderne s'appuie fortement sur des stratégies défensives et par conséquent, marquer un but devient de plus en plus difficile. En utilisant peu de touches de balle par joueur, les attaquants jouent plus vite et ainsi ils peuvent espérer contourner les défenseurs afin de pouvoir tirer au but (Lemoine, Jullien, \& Ahmaidi, 2005). 


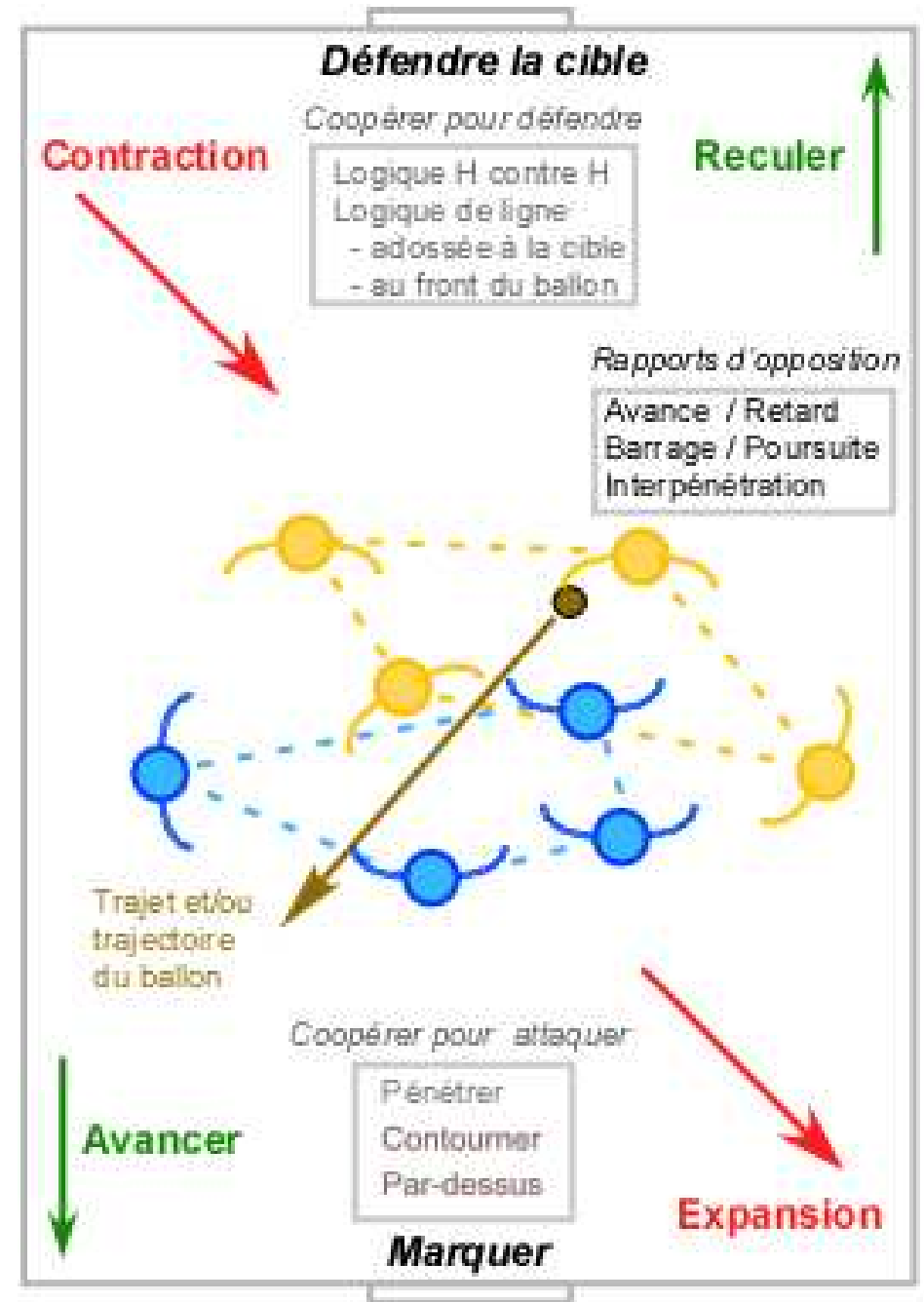

Figure 8. Une modélisation de la dynamique en sport collectif.

Ce modèle dynamique fonctionne dans un espace fini, le terrain, ce qui restreint le champ d'expansion. Une des propriétés de l'affrontement est de présenter une certaine élasticité. Un système élastique est traversé par une série de contractions et d'expansions. Pour les joueurs, il est nécessaire de préserver un état d'équilibre avec peu de temps pour décider alors qu'une tension est appliquée au système. Pour appréhender comment fonctionne l'élasticité du système, nous devons d'abord comprendre comment les configurations du jeu évoluent. Lorsque le système est en forte expansion, il y a des perturbations considérables, de fortes vibrations et des joueurs en mouvement à des vitesses différentes. En forte compression, le système produit des blocages d'où, de ce fait, des difficultés à trouver une réponse adaptée. Aussi, l'élasticité du système produit de l'incertitude. L'incertitude est donc une notion importante qui est fondamentalement liée à la composante temporelle. Un prélèvement rapide et précis d'informations simples rend possible la réduction d'incertitude. Sur le plan tactique, chaque équipe a dès lors pour objectif de réduire le désordre pour elle tout en l'augmentant pour l'équipe adverse. II 


\section{eJRIEPS 26 avril 2012}

résulte de cet ensemble une infinité d'états dynamiques qui rendent compte de l'évolution du rapport d'opposition.

Ici, la délimitation du système concerne à la fois son territoire (une aire de jeu finie), son empan temporel (durée du match, écoulement du temps de jeu) et son objet qualitatif (but du jeu et phénomènes observés). Les joueurs s'organisent en fonction des impératifs des déplacements dans un espace défini. Dans ces trajets, ils s'échangent ou non la balle en fonction de l'évolution de la configuration en cours. La langue de jeu selon Caron et Pelchat (1974) est indispensable à la situation d'apprendre en équipe en vue de la production d'actions en projet. A cet effet, ils proposaient des modèles empiriques et théoriques pour le développement de la tactique en hockey sur glace. En basket-ball, le « contenu approprié », selon Mérand (1990), est aussi « la langue du jeu » permettant aux joueurs de communiquer, de coordonner leurs actions. Pour se déplacer utilement, il faut un plan de circulation (des repères dans l'espace). Pour gérer cette circulation, il faut un code de circulation (orientation, emplacement, déplacement, déviation, accélération ou ralentissement ...). Pour Mérand (1990), toute circulation du ballon et des joueurs découle d'une combinaison entre ces trois éléments :

- l'espace de marque (espace, où le joueur peut envoyer la balle dans la cible) ;

- les déformations de l'EJE et la périphérie du jeu ;

- le couloir de jeu direct (défini par le couloir qui va du ballon à la cible à tout moment).

La décomposition en sous-systèmes a bien consisté à définir des ensembles donnant lieu à des sous-modèles relativement autonomes tout en respectant les propriétés essentielles du système d'affrontement.

Dans ce contexte, Bouthier (1989) souligne l'intérêt du concept de référentiel commun pour expliciter les formes, les conditions et les moyens de la coordination individuelle des actions. Le référentiel commun, au-delà d'un premier niveau basé sur la logique du règlement, correspondrait à une véritable méthodologie d'analyse du rapport d'opposition et de décision d'orientation des actions. Bouthier (2000) met également en avant l'importance de ce référentiel en ce qui concerne l'articulation des aspects stratégiques et tactiques en vue d'assurer, si possible, la pertinence et la compatibilité des choix du point de vue de l'évolution du jeu.

Enfin, les deux opérations effectuées (étude théorique et délimitations) en vue de modéliser sont interdépendantes et l'on peut toujours modifier les frontières du système en 
eJRIEPS 26 avril 2012

modifiant la place de certains sous-systèmes de façon à faciliter l'étude de l'affrontement en la rendant plus fiable et plus cohérente.

\section{Conclusion}

La succession des déformations de l'espace de jeu effectif et l'évolution des configurations du jeu ainsi que l'élasticité inhérente aux formes du jeu constituent des indicateurs privilégiés de l'affrontement. Les joueurs ne sont plus conçus comme liés à un espace fixe, mais comme des mobiles occupant l'espace de jeu dans un cadre de potentialités (Fernandez, 2002).

Ainsi, l'objectif de cette réflexion était de discuter la théorie des systèmes dynamiques et son utilisation directe dans le champ de l'étude des sports collectifs, tout en proposant de synthétiser et/ou de créer des outils conceptuels plus pertinents pour analyser des phénomènes évolutifs, complexes. Dans les sports collectifs, à partir d'une trame de jeu connue, en fonction de l'importance de la rencontre, de l'évolution du score, de l'écoulement du temps de jeu et de leur perception du rapport de forces, les joueurs peuvent, dans certaines limites, changer ou faire évoluer la stratégie prévue par l'adoption de tel ou tel type de comportement qui renvoie à des choix tactiques différents. Ici, les modélisations proposées montrent toute leur importance pour décider juste et vite.

Ce travail se situe en premier lieu dans une perspective d'analyse de la dynamique dans les sports collectifs. Une des facettes de cette dynamique suppose que le comportement des systèmes converge vers des états qui tendent à présenter parfois une certaine stabilité dans le temps. Par ailleurs, le match est par définition ouvert, soumis à des influences extérieures et sa dynamique est aussi dépendante des fluctuations liées d'une manière proche et nécessaire au fonctionnement des sous-systèmes (le match, l'affrontement partiel ou encore le un contre un). Sans de telles analyses, des informations significatives ne seraient pas mises à la disposition des chercheurs, des enseignants ou des entraîneurs. Nous pensons que cette approche de la dynamique en sport collectif est essentielle et il est bien évident que ce type de conception doit avoir des conséquences sur l'entraînement, la recherche ou l'apprentissage en proposant des situations dynamiques où la pression temporelle est majeure.

Enfin, l'ensemble de ces modèles constitue une réflexion qui est à enrichir, à discuter et à faire évoluer pour que les conceptions et l'analyse du jeu soient de plus en plus fiables et pertinentes. 


\section{eJRIEPS 26 avril 2012}

\section{Bibliographie}

Almond, L. (1986). Reflection on themes: A game classification. In R. Thorpe, D. Bunker, \& L. Almond (Eds.), Rethinking games teaching (pp. 71-72). Loughborough, England: Loughborough University of Technology.

Brackenridge, C. (1979). Games: Classification and analysis. Conférence présentée aux Kirkless Teachers, le 02 mai 1979.

Bourdieu, P. (1972). Esquisse d'une théorie de la pratique. Genève : Droz.

Bouthier, D. (1989). Les conditions cognitives de la formation d'actions sportives collectives. Le Travail Humain, 52, 175-182.

Bouthier, D. (2000). La coordination des décisions individuelles ; contribution de l'intelligence tactique. Colloque Préparation Olympique L'évolution de la pensée tactique, Noisy le Grand, 14 et 15 novembre 2000.

Caron, J., \& Pelchat, C. (1974). Le Hockey, sport collectif : modèle empirique ou théorique. Mouvement, 9(1), 33-46.

Caty, D., Meunier, J.N., \& Gréhaigne, J.F. (2007). Modélisations des attaques réussies pour progresser dans les sports collectifs en EPS. Spirales, 40, 105-116.

Conquet, P., \& Devaluez, J. (1986). Conception de l'éducation physique. In SNEP (Ed.) E.P.S. contenus et didactique (pp. 91-103.). Paris : SNEP.

Davids, K., Araújo, D., \& Shuttleworth, R. (2005). Applications of dynamical systems theory to football. In T. Reilly, J. Cabri, \& D. Araújo (Eds.), Science and football $V$ (pp.537- 550). London: Routledge, Taylor \& Francis.

Davids, K., Glazier, P., Araujo, D., \& Bartlett, R. (2003). Movement systems as dynamical systems. The functional role of variability and its implications for sports medicine. Sports Medicine, 33, 245-260

Deleplace (1966). Le rugby. Paris : Colin- Bourrelier.

Deleplace, R. (1979). Rugby de mouvement - Rugby total. Paris: Éducation Physique et Sport.

Deleplace, R. (1994). La notion de matrice d'action pour les actions motrices complexes. In D. Bouthier \& J. Griffet (Eds.), Représentation et action en activité physique et sportive (pp. 25-42). Paris : Université de Paris-Orsay.

Dugrand, M. (1989). Le football : de la transparence à la complexité. Paris : PUF.

Fernandez, A. (2002). Rupture et prise d'avantage en sports collectifs, un modèle théorique. eJournal de la Recherche sur l'Intervention en Education Physique et sportive, 2, 03-17. 


\section{eJRIEPS 26 avril 2012}

Frencken, W., Lemmink, K., Delleman, N., \& Visscher, C. (2011). Oscillations of centroid position and surface area of soccer teams in small-sided games. European Journal of Sport Science, 11, 215-223.

Gentner, D., Holyoak, K., \& Kokinov, B. (Eds.). (2001). The analogical mind: Perspectives from cognitive science. Cambridge, MA: MIT Press.

Gréhaigne, J.F. (1989). Football de mouvement. Vers une approche systémique du jeu.

Thèse de Doctorat (non publiée). Université de Bourgogne, Dijon, France.

Gréhaigne, J.F. (1992). L'organisation du jeu en football. Paris : ACTIO.

Gréhaigne, J.F. (Ed.). (2007). Configurations du jeu, débat d'idées et apprentissage des sports collectifs. Besançon: Presses de l'Université de Franche-Comté.

Gréhaigne, J.F. (Ed.). (2009). Autour du temps. Espaces, apprentissages, projets dans les sports collectifs. Besançon : Presses de l'Université de Franche-Comté.

Gréhaigne, J.F. (Ed.). (2011). Des signes au sens. Le jeu, les indices, les postures et les apprentissages dans les sports collectifs à l'école. Besançon: Presses de l'Université de Franche-Comté.

Gréhaigne, J.F., Billard, M., \& Laroche, J.Y. (1999). L'enseignement des jeux sportifs collectifs à l'école. Conception, construction, évaluation. Bruxelles : De Boeck.

Gréhaigne, J.F., \& Bouthier, D. (1994). Analyse des évolutions entre deux configurations du jeu en football. Science et Motricité, 24, 44-52.

Gréhaigne, J.F., Bouthier, D., \& David, B. (1997). Dynamic systems analysis of the opponent relationships in the collective actions in soccer. Journal of Sports Sciences, 15, 137-149.

Gréhaigne, J.F., Caty, D., \& Marle, P. (2004). L'apport de la notion de configuration du jeu à la didactique des sports collectifs. In G. Carlier (Ed.), Si l'on parlait du plaisir d'enseigner l'éducation physique (pp. 167-179). Montpellier : AFRAPS.

Gréhaigne, J.F., Godbout, P., \& Bouthier, D. (1999). The foundations of tactics and strategy in team sports. Journal of Teaching in Physical Education, 18, 159-174.

Gréhaigne, J.F., Godbout, P., \& Zerai, Z. (2011). How the "rapport de forces" evolves in a soccer match: The dynamics of collective decisions in a complex system. Revista de Psicología del Deporte, 20, 747-764.

Jeu, B. (1977). Le sport, l'émotion, l'espace : essai sur la classification des sports et ses rapports avec la pensée mythique. Paris : Vigot 


\section{eJRIEPS 26 avril 2012}

Lemoine, A., \& Jullien, H. (2008). Jeu en déviation et configurations du jeu en football. e Journal de la Recherche sur l'Intervention en Education Physique et sportive, 15, 05-20.

Lemoine, A., Jullien, H., \& Ahmaidi, S. (2005). Technical and tactical analysis of one-touch playing in soccer - Study of the production of information. International Journal of Performance Analysis in Sport, 5, 83-103.

McGarry, T., Anderson, D., Wallace, S., Hughes, M., \& Franks, I. (2002). Sport competition as a dynamical self-organizing system. Journal of Sports Sciences, 20, 771-781.

Moniotte, J., Nadeau, L., \& Fortier, K. (2011). Configurations de jeu d'équipes de hockey sur glace de niveau Pee Wee et Bantam. e Journal de la Recherche sur I'Intervention en Education Physique et sportive, 24, 31-52.

Mouchet, A. (2003). Caractérisation de la subjectivité dans les décisions tactiques des joueurs d'Elite 1 en rugby. Thèse de Doctorat non publiée, Université Bordeaux 2, France. Site www.expliciter.net

Mérand, R. (1977) L'éducateur face à la haute performance olympique. Paris, France: Sport et Plein air.

Mérand, R. (Ed.). (1990). Basket-ball : lancer ou circuler ? Rencontres Pédagogiques, 28, 9-38.

Planck, M. (1941). Initiation à la physique. Paris : Flammarion.

Walliser, B. (1977). Systèmes et modèles. Introduction critique à l'analyse de systèmes. Paris : Le Seuil.

Zerai, Z. (2011). Apprentissage du handball chez les jeunes filles Tunisiennes et Françaises ; apport de la verbalisation. Thèse (non publiée) en Science du Sport. Université de Franche-Comté.

Ziane, R. (2004). Contribution à la formation des entraîneurs sportifs. Caractérisation et représentation des actions de jeu : l'exemple du basket-ball. Thèse de Doctorat (non publiée), École Normale Supérieure de Cachan. 